\title{
Comparative Study of Kernel Pseudo-Error Monitor
}

\author{
Wang Peng, Wu Zhengyang, Qian Feng
}

School of Physical and Electronic Engineering, Changshu Institute of Technology, Jiangsu Province, China, 215500

\author{
Email: epwang@xatu.edu.cn
}

Keywords: Symbol Error Rate; Pseudo-Error Monitor; Kernel Density Estimation

\begin{abstract}
The symbol error rate is the ultimate measure of the reliability of a data transmission service. However, due to the absence of effective and efficient solutions, the symbol error rate estimation has been long neglected in practice. Kernel pseudo-error monitoring represents the latest effort to fill the gap. The paper demonstrates the advantages of the method by comparing its performance to other solutions in various conditions.
\end{abstract}

\section{Introduction}

The symbol error rate (SER) is widely recognized as the ultimate measure of the reliability of a data transmission service. While theoretical SER analysis, or called model-based SER estimation, has been studied extensively in literature, model-independent BER estimation has received much less attention, probably due to the absence of effective and efficient solutions.

The simplest model-independent method is to compare the received message to the transmitted and enumerate the transmission errors [1]. Apparently, the effectiveness of the method is heavily dependent on the prior knowledge of the transmission. The technique of pseudo-error monitoring overcomes this problem by extrapolating the SER from a set of pseudo-error rates estimates [2,3]. Pseudo-error monitoring is also advantageous in reducing the observation time for maintaining reliable estimation. However, the observation cost is still unbearable for practical applications. In pursuit of maximum utilization of the observations and thus least observation cost, the strengths of kernel density estimation have been incorporated into SER estimation, and leads to the so-called kernel real- and pseudo-error monitoring [4]-[7].

Kernel pseudo-error monitoring (KPEM) is expected to be more reliable than kernel real-error monitoring (KREM), especially in blind SER estimation, i.e., when the knowledge of the transmission is unavailable. However, this statement is never substantiated in literature. The paper compares the performances of both solutions in various environments. The simulation results clearly show the advantages of KPEM. Besides, a general framework for kernel-based SER estimation is presented and a short analysis of the blind incapability of KREM is also given.

\section{Kernel-Based SER Estimation}

In general, a kernel SER estimator for an $M$-ary communication system can be expressed as

$$
\hat{P}=\sum_{m}\left[P_{m} \cdot \int_{\varepsilon_{m}} \hat{f}_{m}\left(x_{m}\right) d x_{m}\right]
$$

wherein $P_{m}$ is the probability that the $m$ th symbol is transmitted, $x_{m}$ represents the corresponding decision statistic, $\varepsilon_{m}$ represents the error region of $x_{m}$, and $f_{m}$ is the probability density function (PDF) of $x_{m}$. The kernel estimator of $f_{m}\left(x_{m}\right)$ is given by

$$
\hat{f}_{m}\left(x_{m}\right)=\frac{1}{n_{m} h} \sum_{i=1}^{n_{m}} K\left(\frac{x_{m}-X_{m i}}{h}\right)
$$

where $X_{m i}, i=1, \cdots, n_{m}$, denotes the $i$ th observation of $x_{m}, \Sigma_{m} n_{m}=n, h$, the smoothing parameter, 
is a positive value that determines the amount of the details to be masked in the approximation, and $K(\cdot)$, the kernel function, defines the method of smoothing the observations. Usually, the standard Gaussian function is employed as the kernel.

Under the assumption that the $M$ symbols are transmitted with equal probability, and $n$ is sufficiently large such that $n_{m} / n$ approaches $M^{-1}$, Eq. (1) becomes

$$
\hat{P}=\frac{1}{n} \sum_{m=0}^{M-1} \sum_{i=1}^{n_{m}} T\left(r_{m l}, r_{m u}, X_{m i}\right)
$$

where $r_{m l}$ and $r_{m u},-\infty \leq r_{m l}<r_{m u} \leq \infty$, are respectively the lower and the upper decision thresholds of the $m$ th symbol, and $T(\cdot)$ denotes the tails of the kernel. For standard Gaussian kernel, it is given by

$$
T(\cdot)=Q\left(\frac{X_{m i}-r_{m l}}{h}\right)+Q\left(\frac{r_{m u}-X_{m i}}{h}\right) .
$$

The kernel SER estimate can therefore be interpreted as the mean coverage of the tails of the kernel. Each tail reveals the error probability of a specific observation of the $m$ th symbol.

In KREM, Eq.(3) is applied directly to SER estimation. Without the knowledge of the source, the KREM cannot properly classify the observations of different symbols. As a result, the target of PDF estimation is not the real density function but a distorted version of it with tail(s) folded, partially or fully. Such a deviation is trivial for PDF estimation since the tail is negligible compared to the main body, but for SER estimation it can be easily a disaster. Figure 1 clarifies this problem, where $f, f_{B}$ and $\hat{f}_{B}$ represent respectively the real, the distorted and the estimated density functions.

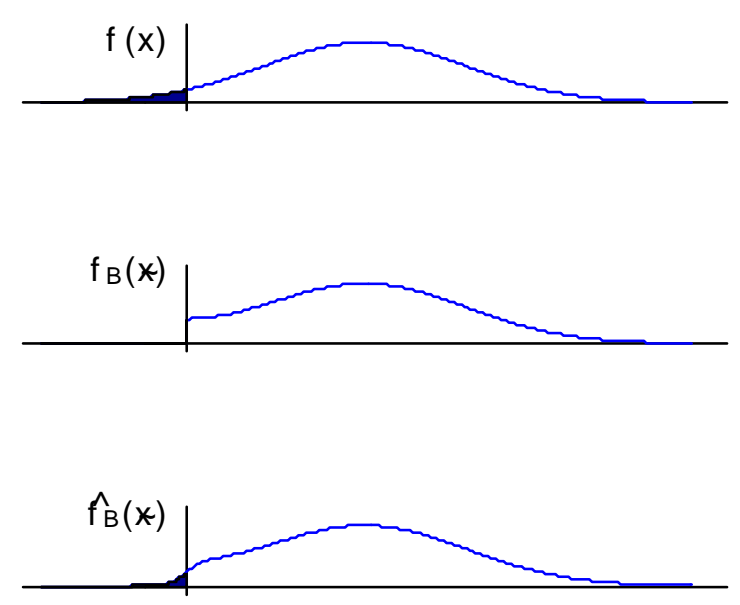

Fig. 1. KREM in blind SER estimation

In KPEM, the SER is purposely amplified to release the demand on the length of the observation sequence, and Eq. (3) is used to calculate a set of pseudo-error rates, which are then extrapolated to approach the SER (refer to [7] for the details of KPEM).

\section{Computer Simulations}

Computer simulations have been conducted to compare the performance of KPEM, KREM and traditional pseudo-error monitoring, TMM (threshold-modification monitoring). In Figure 2 and 3, the three estimators are compared by, respectively, the estimation error and the percent coverage for tolerance level $[0.2 P, 5 P]$, where Gaussian channel is assumed, the KREM takes advantage of the knowledge of the transmitted signal and the other two estimators work totally in blind state. 


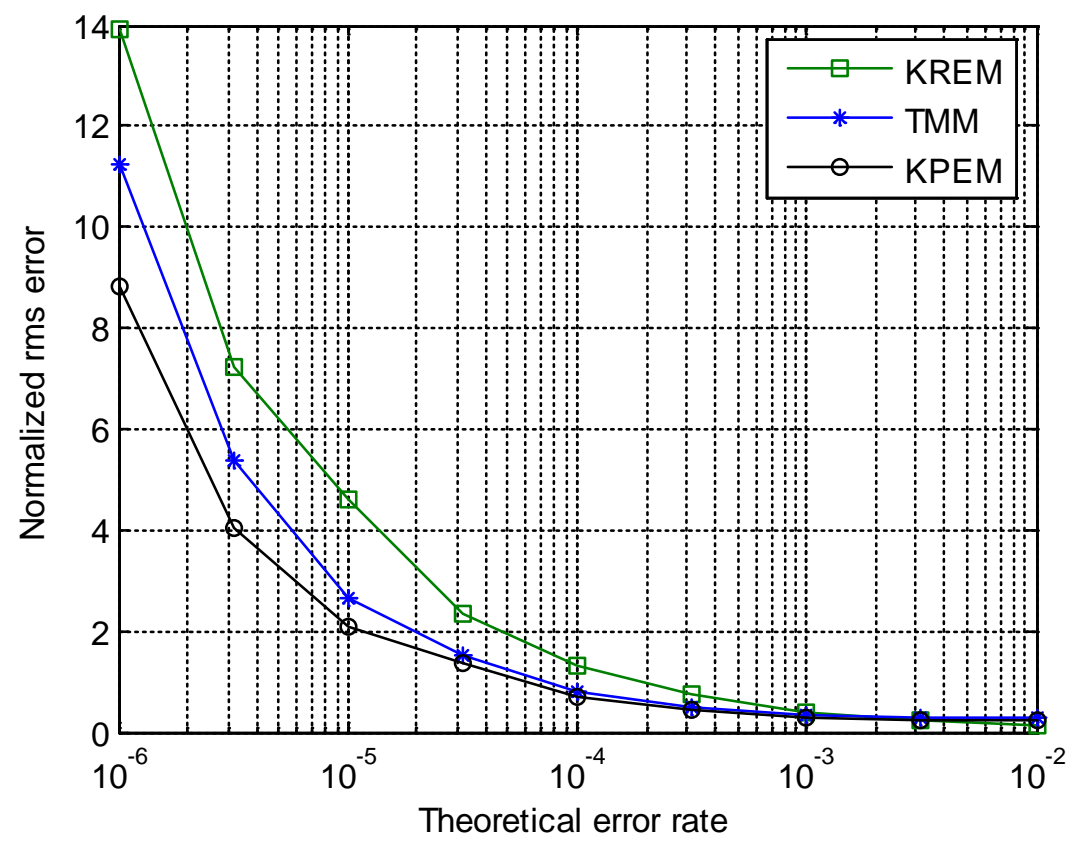

Fig. 2. Comparison of SER estimators by the estimation error

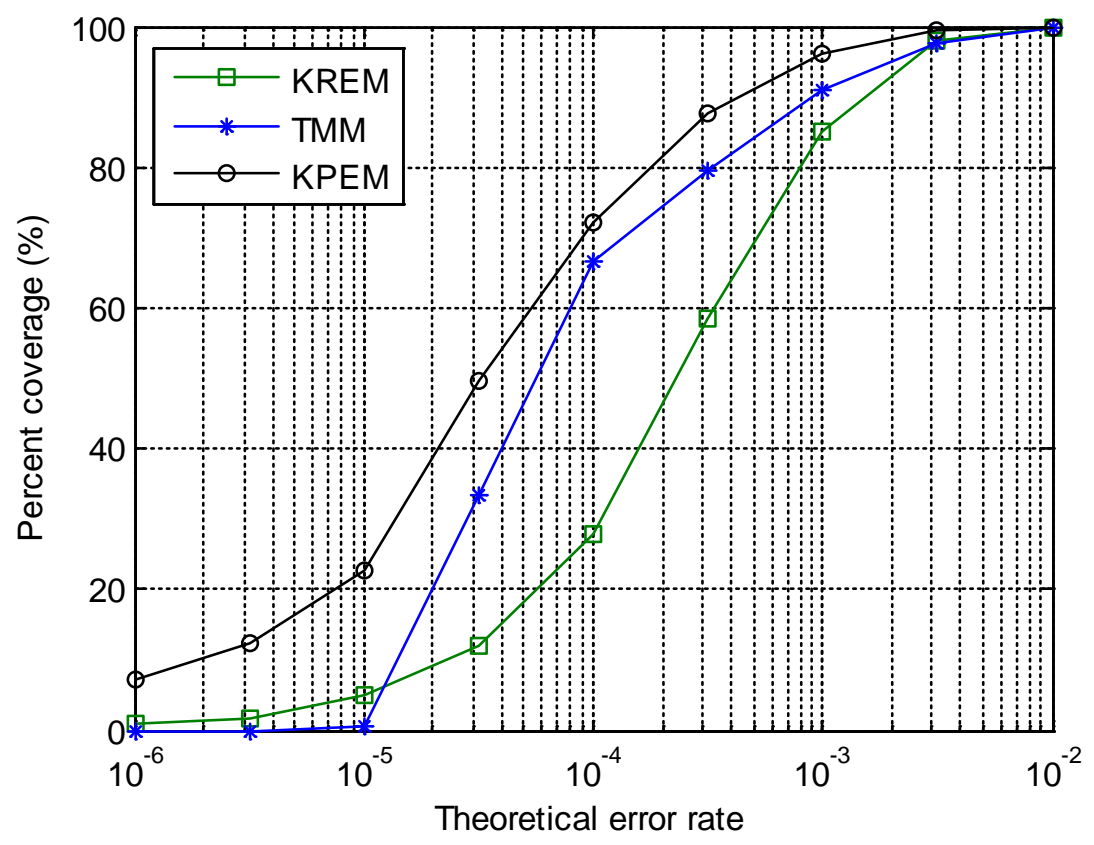

Fig. 3. Comparison of SER estimators by the percent coverage

As can be seen, KPEM combines the strengths of KREM and TMM and gives more accurate and robust estimation. Figure 4 further compares KPEM with KREM by simply presenting their estimation results obtained at different noise levels, wherein the dotted lines denote theoretical SERs. Note that when the SER is relatively low, the KREM may fail to collect sufficient transmission errors to perform valid estimation and give trivial result of zero. For non-Gaussian channels, the simulation results are quite similar and are thus excluded from the paper. 

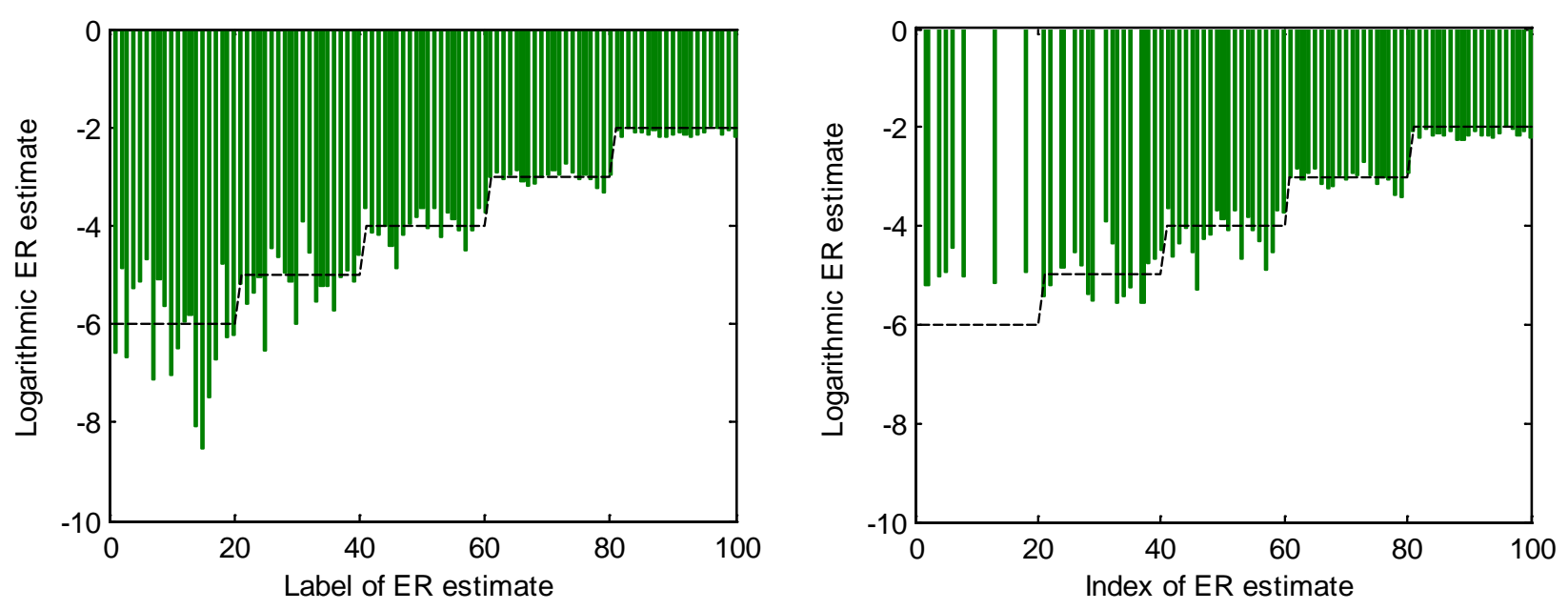

Fig. 4. SER estimates produced by KPEM (left) and KREM (right)

\section{Conclusion}

A comparative study on KPEM has been conducted, and the simulation results presented in the paper demonstrate that KPEM gives much better performance than KREM in blind SER estimation. Moreover, KPEM is evidently more effective in reducing the observation cost.

\section{References}

[1] E. A. Newcombe and S. Pasupathy, "Error rate monitoring for digital communications," Proc. IEEE, vol. 70, no. 8. pp. 805-822, 1982.

[2] D. J. Gooding, "Performance monitor technique for digital receivers based on extrapolation of error rate,” IEEE Trans. Commun. Technol., vol. 16, no. 3, pp. 380-387, 1968.

[3] J. M. Keelty and K. Feher, "On-line pseudo-error monitors for digital transmission systems," IEEE Trans. Commun., vol. COM-26, no. 8, pp. 1275-1282, 1978.

[4] T. J. Nohara, A. Premji, and W. R. Seed, “A new signal quality degradation monitor for digital transmission channels,” IEEE Trans. Commun., vol. 43, no. 2, pp. 1333-1336, 1995.

[5] J. D. Laster, J. H. Reed, and W. H. Tranter, "Bit error rate estimation using probability density function estimators,” IEEE Trans. Veh. Technol., vol. 51, no. 1, pp. 260-267, 2003.

[6] K. A. Phillips, J. H. Reed, and W. H. Tranter, “Minimum BER adaptive filtering,” in Proc. IEEE Int. Conf. Commun., pp. 1675-1680, New Orleans, LA, Jun. 2000.

[7] P. Wang and W. Ser, “A novel pseudoerror monitor,” EURASIP J. Appl. Signal Process., vol. 2004, no. 3, pp. 412-417, 2004. 